\title{
3-D Spectral IP Imaging: Non-Invasive Characterization DE FG02 96ER 14714
}

\section{Summary}

The Earth Resources Laboratory (ERL) performed a broad foundational study of spectral induced polarization (SIP) for site characterization. The project encompassed laboratory studies of microgeometry and chemistry effects on Induced Polarization (IP), an investigation of electromagnetic coupling (emc) noise, and development of 3D modeling and inversion codes. The major finding of the project is that emc noise presents a critical limitation for field implementation of SIP and conventional correction methods are inadequate. The project developed a frequency domain 3D complex resistivity modeling and inversion code

Laboratory experiments were conducted to study the effects of solution chemistry and microgeometry on the SIP response of sandstone. Results indicate that changes in chemistry affect the magnitude of the spectral IP response and changes in microgeometry affect the shape of the spectral IP response. The developed physiochemical IP model can be used to invert spectral IP data for an apparent grain size distribution.

Laboratory studies over the last twenty years have shown that SIP data must be acquired over several decades of frequency and include frequencies greater than $1 \mathrm{kHz}$. A model of the components of emc noise has been developed and investigation with this model showed that inductive coupling is the most significant component. The study concluded that emc limits the frequency range of usable field data to approximately $100 \mathrm{~Hz}$ and below for typical site conditions. Several correction schemes have been developed based on treating emc as noise to be removed from the data, but our investigation has shown that these are not adequate for high frequencies, greater than $100 \mathrm{~Hz}$. Laboratory studies have demonstrated that the greatest response is the frequency range greater than $1 \mathrm{KHz}$, hence the emc problem must be resolved for field implementation of SIP to advance.

The ERL developed 2D/3D time domain codes that perform inversions for chargeabilities based on schemes introduced by Siegel (1959). The ERL has also developed a 3D complex resistivity code for inversion of frequency domain IP data. The algorithm accommodates a general earth model with a complex electrical resistivity as a function of frequency and 3-D spatial position. The forward problem is solved by the complex biconjugate gradient method, while the regularized inverse problem is solved by the nonlinear conjugate gradient method.

Time domain field data was acquired along a single survey line at the FS-12 plume, Massachusetts Military Reservation. The data was inverted with the developed 2D time domain code with the results having an excellent match to monitoring well data. The data was further analyzed by an innovative scheme where a "gross spectral chargeability" was determined from time domain data. The results show that there is valuable information in the spectra of the data. 